\title{
Room temperature negative differential conductance due to resonant tunneling through a single nanocrystalline-Si quantum dot
}

\author{
Akhmadi Surawijaya $^{1}$, Hiroshi Mizuta ${ }^{1,2}$ and Shunri Oda ${ }^{1,2}$ \\ ${ }^{1}$ Department of Physical Electronics and Quantum Nanoelectronics Research Center, Tokyo Institute of Technology \\ 2-12-1 O-okayama, Meguro-ku, Tokyo 152-8552, Japan \\ Phone: +81-3-5734-2542 E-mail: akhmadi@ neo.pe.titech.ac.jp \\ ${ }^{2}$ SORST-JST (Japan Science and Technology), 4-1-8 Honmachi, Kawaguchi, Saitama 332-0012, Japan
}

\section{Introduction}

For the last two decades resonant tunneling has been studied extensively for compound semiconductor multiple barrier structures [1],[2]. Recent development has allowed resonant tunneling phenomena to be observed in a-Si:H/a-Si $\mathrm{N}_{4}: \mathrm{H}$ [3] and $\mathrm{CaF}_{2} / \mathrm{CdF}_{2}$ [4] double barrier system. Negative differential conductance (NDC) in Si double barrier structure due to resonant tunneling has been observed [5], [6]. Room temperature NDC in a self-assembled $\mathrm{Si}$ quantum dot has also been reported [7].

Strong confinement effect in a Si quantum dot (QD) is the key to realize operation of resonant tunneling device at room temperature. In this work we adopted a nanocrystalline-Si (nc-Si) QD deposited by UHV-PECVD system. The nc-Si dot is covered with a thin $\mathrm{SiO}_{2}$ layer of about $2 \mathrm{~nm}$ in thickness (Figs. 1 and 2). We characterized tunneling current through a single nc-Si QD by using the contact mode AFM. We also performed 3D scattering matrix calculation [8] to analyze the device transmission probability spectrum and the electron wave function at resonance.

\section{Sample preparation}

We used n-type $\langle 100\rangle \mathrm{Si}$ wafer for the substrate with $1 \times 10^{-3}-2 \times 10^{-2} \Omega \mathrm{cm}$. After $\mathrm{H}_{2} \mathrm{SO}_{4}: \mathrm{H}_{2} \mathrm{O}_{2}$ and $\mathrm{HF}$ cleaning, 2 $\mathrm{nm}$ chemical oxide was grown by $\mathrm{H}_{2} \mathrm{SO}_{4}: \mathrm{H}_{2} \mathrm{O}_{2}$ (30:70) at $130^{\circ} \mathrm{C}$ for 10 minutes. A single layer of nc-Si dot was then deposited on the sample by using UHV-PECVD using pulses of silane and Ar gas. The top oxide barrier was formed by oxidation of the dot at room temperature. Typical native oxide thickness is 1.5 to $2 \mathrm{~nm}$. The back contact was formed with silver paste, and the gold coated AFM cantilever tip was used for the top contact.

The distribution and size of the nc-Si dots were observed by SEM (Fig. 2). The dots are sparsely distributed on the substrate with a surface density of. $1.4 \times 10^{-8} \mathrm{~cm}^{2}$ and average dot diameter of $10 \mathrm{~nm}$. Individual dots are single-crystalline $\mathrm{Si}$ as shown in the HRTEM image in the inset to Fig. 2.

For the contact-AFM measurement we used Seiko SPA-400 unit and SPI3800 probe station. For the cantilever we used the SI-AF01-A with gold coated tip. We performed contact-AFM scanning with $1 \times 1 \mu \mathrm{m}^{2}$ scan area to generate the topography image. The AFM measurement was performed by selecting a single nc-Si QD from the topographical view. Due to the drift in AFM image, a topographical scan must be conducted every time before we measure the current via the nc-Si QD.

\section{Room temperature I-V characteristics}

$\mathrm{I}-\mathrm{V}$ characteristics were measured for the single nc-Si dot at room temperature. Figure 3 shows the typical I-V curve observed when the sample was positively biased from 0 to $4.5 \mathrm{~V}$. Two current peaks were clearly observed at room temperature: the first peak around $1 \mathrm{~V}$ and the second one around $2.5 \mathrm{~V}$. The largest peak-to-valley $(\mathrm{P} / \mathrm{V})$ current ratio observed is 17 for the $1^{\text {st }}$ resonance.

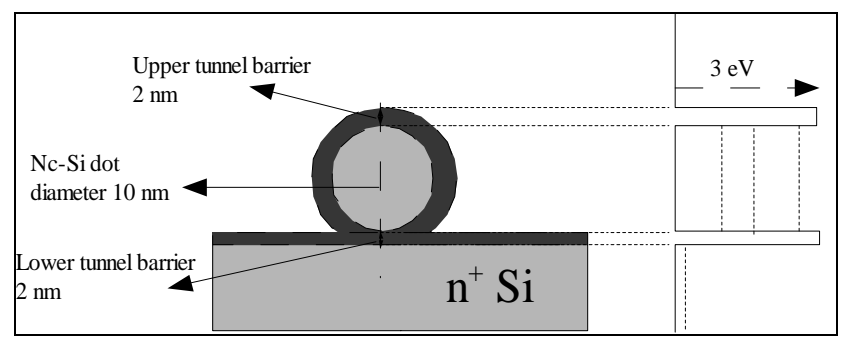

Fig. 1 Schematic diagram of a single nc-Si QD resonant tunneling structure covered with a thin $\mathrm{SiO}_{2}$ tunnel barrier.

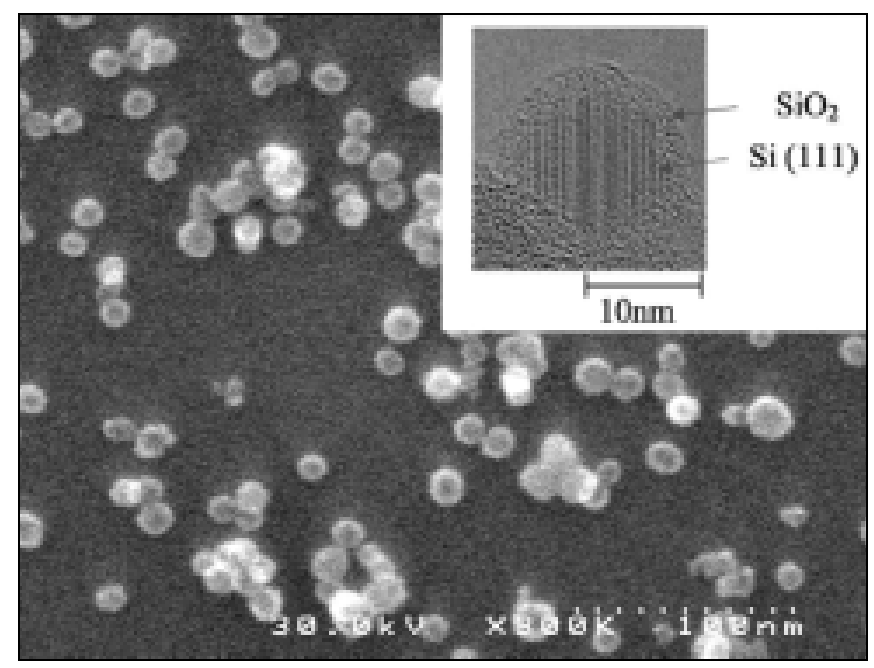

Fig. 2 SEM image of the nc-Si dot deposited on the chemically grown $\mathrm{SiO}_{2}$ film. The inset shows the HRTEM image of a single nc-Si QD.

\section{3D S-matrix simulation of transmission spectrum}

The single nc-Si QD structure used for simulation is shown in Fig. 4. It consists of an undoped Si QD with diameter of $10 \mathrm{~nm}$ embedded in $\mathrm{SiO}_{2}$ matrix, which is sand- 
wiched between 2-nm-thick heavily-doped Si contact layers. Spacing between the contact region and the edge of the QD is $1 \mathrm{~nm}$. Si/SiO ${ }_{2}$ conduction band discontinuity is assumed to be $3.0 \mathrm{eV}$. The entire structure was discretized by using 3D finite difference mesh, and the 3D Schrödinger equation with an open boundary condition was solved by using a 3D scattering matrix formula [8]. The calculated total transmission spectrum is shown in figure 5. The associated 3D probability density of electron calculated at the first resonance for the first incident wave from the emitter is shown in Fig. 6, an orthoslice taken at the center of the dot showing a spherical nature of the first quasi-bound state of the QD.

\section{Conclusion}

Room temperature NDC has been observed in a single nc-Si QD RTD. The largest PVR is 17 in room temperature. The current peaks are attributable to resonant tunneling through the quasi bound states of the nc-Si QD.

\section{Acknowledgements}

The author would like thank K. Usami for the nc-Si dot deposition, Y. Tsuchiya and S. Huang for the discussions.

\section{References}

[1] R.Tsu and L. Esaki, Appl. Phys. Lett., 22 (1973). 562,

[2] L.L. Chang, L. Esaki, and R. Tsu, Appl. Phys. Lett., 24 (1974) 593.

[3] S. Miyazaki, Y. Ihara and M. Hirose, Phys. Rev. Lett., 59 (1987) 125.

[4] M. Watanabe, T. Funagawa, T. Teraji and N. Sakamaki, Jpn. J. Appl. Phys. Lett., 39 (2000) 716.

[5] Y. Ishikawa, T. Ishihara, M. Iwasaki and M. Tabe, Electronics Letter, 37 (2001) 1200.

[6] H. Ikeda, M. Iwasaki, Y. Ishikawa and M.Tabe, Appl. Phys. Lett., 83 (2003) 1456.

[7] M. Fukuda, K. Nakagawa, S. Miyazaki and M. Hirose, Appl. Phys. Lett, 70 (1997) 2291.

[8] H. Mizuta and T. Tanoue, The Physics and Applications of Resonant Tunnelling Diodes, Cambridge University Press (1995).

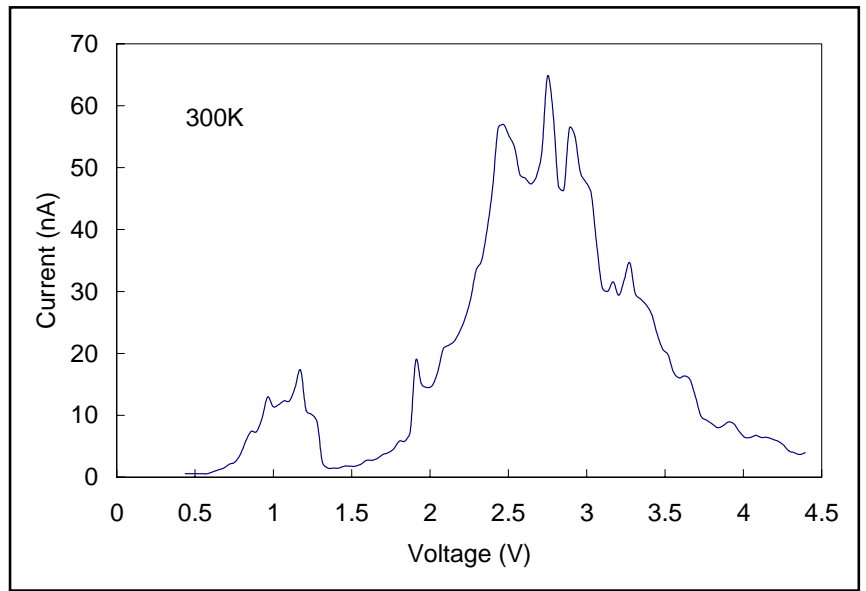

Fig. 3 Room temperature I-V characteristics with double NDC.

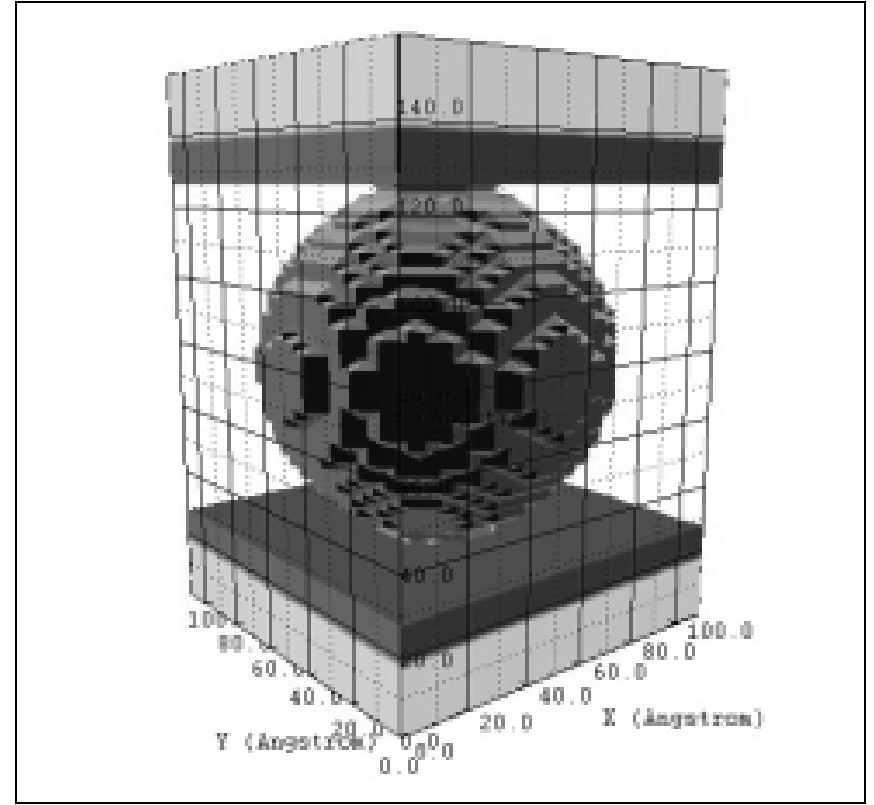

Fig 4 3D structure of the nc-Si dot sandwiched between two oxide barriers used for simulation.

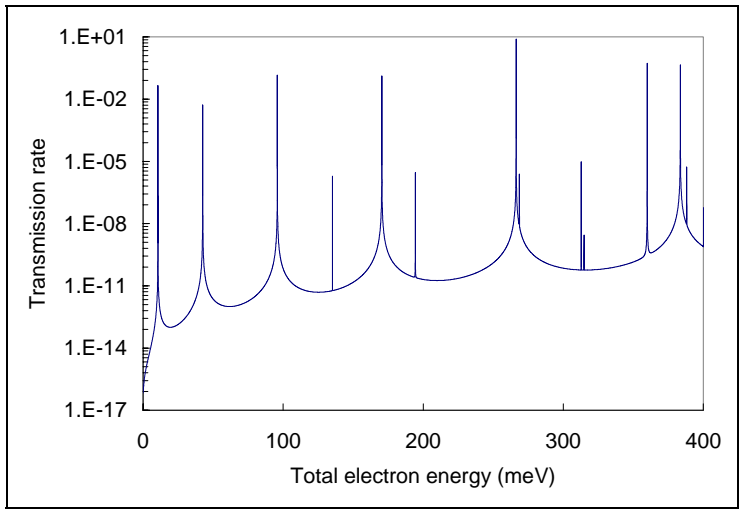

Fig. 5 Transmission rate calculated for a single nc-Si QD RTD.

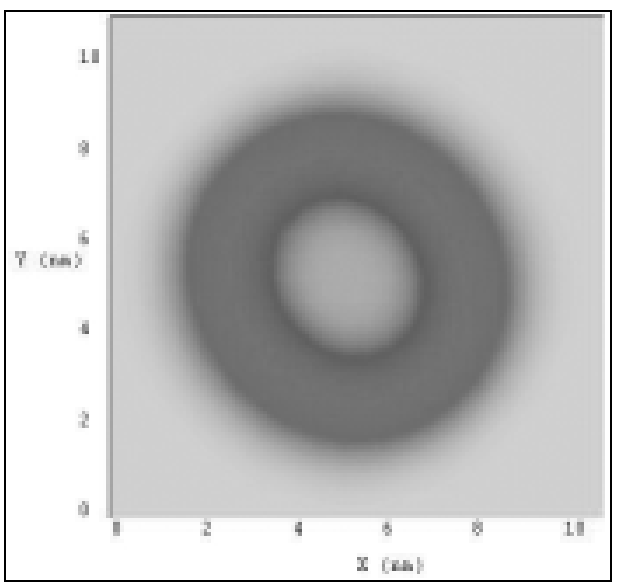

Fig. $63 \mathrm{D}$ probability density of electron at the center of the dot calculated for the incident wave from the emitter with the lowest lateral mode. 